\title{
Transcriptomics as a Tool to Monitor and Predict the Behaviour of the Microbiome in an Industrial Salt Waste Water Treatment Plant
}

\author{
G.J.W. Euverink ${ }^{1}$, A.K. Geurkink1, 3, G. Faber ${ }^{3}$, V. Rijks ${ }^{3}$, E. Croese ${ }^{3}$, I. Dinkla ${ }^{3}$, P. van der Marel ${ }^{4}$, J. \\ Krooneman $^{1}$, B. Jayawardhana ${ }^{2}$ \\ ${ }^{1}$ Products and Processes for Biotechnology, Engineering and Technology institute Groningen \\ University of Groningen \\ The Netherlands \\ ${ }^{2}$ Discrete Technology and Production Automation, Engineering and Technology institute Groningen \\ University of Groningen \\ ${ }^{3}$ Bioclear b.v. \\ The Netherlands \\ ${ }^{4}$ North Water b.v. \\ The Netherlands
}

In waste water treatment plants polluted water is purified with the help of complex ecosystems of many different bacteria. In these systems many different desirable but also undesirable microbial activities play a role. To achieve detailed insight in the microbial activities of these processes in a waste water treatment plant metagenomic tools can be applied. Until now transcriptomics tools are almost exclusively reserved for applications in fundamental research. One of the reasons is that metagenomic techniques are complex and generates a very large dataset. Furthermore, in most cases it takes too long to obtain practical answers and solutions to questions raised in the 'real world'. The costs associated with such an approach are also very high compared to the costs of measuring a set of standard physical and chemical parameters. However, transcriptomics results in an enormous amount of knowledge about the microbial processes in a waste water treatment plant that can be used to improve the quality of the effluent. Therefore, new pragmatic ways to bridge the gap between transcriptomics and business are required. This approach is characterized by the strong cooperation between fundamental research and a practical application. It distinguishes itself by combining Next Generation Sequence (NGS) data of DNA and RNA extracted from a waste water treatment plant at specific time points. NGS data will give detailed insight into the population and activities of a sample while (high throughput) quantitative-PCR is used to get insight into the dynamics of selected set of target genes. Mathematical analysis of the historical data combined with the chemical/physical characteristics of the influent water allows us to predict and control the microbial behavior and thereby the performance of the waste water treatment plant. 